\title{
Representações sociais do cuidado ao idoso e mapas de rede social
}

\author{
Social representations of elderly people care and social network maps
}

\author{
Annie Mehes Maldonado Brito, ${ }^{\mathrm{a},}$, Brigido Vizeu Camargo ${ }^{\mathrm{a}}$, \\ Andréia Isabel Giacomozzi ${ }^{\mathrm{a}}$, Bruna Berri ${ }^{\mathrm{a}}$ \\ aUniversidade Federal de Santa Catarina, Brasil
}

\begin{abstract}
Resumo
Este estudo teve como objetivo compreender as representações sociais de idosos acerca dos cuidados para consigo, para com outros idosos e relativas à sua rede social, relacionando-as com práticas de cuidados dispensados pela rede social. O estudo compreendeu um levantamento de dados com 102 participantes idosos, a partir do sexo e do grau de dependência, com os objetivos de identificar a rede social do idoso e a parte estrutural da representação social do cuidado do idoso. Os resultados evidenciados pela configuração e o mapa de redes indicaram diferença na configuração da rede social do idoso entre os sexos, com os homens e os idosos independentes em menor condição de solidão, o que pode evidenciar uma leve deterioração da rede social do idoso dependente. Na prática social o cuidado ao idoso é delegado ao cuidador informal, familiar e do sexo feminino. A representação social do cuidar da pessoa idosa envolveu como marca principal os aspectos pragmáticos do cuidado e sua funcionalidade, bem como a passividade do mesmo. Verificou-se a necessidade de políticas de educação para a saúde e o envelhecimento que privilegiem não somente os idosos, mas também as pessoas que se aproximam da vivência da velhice, em especial para aquelas que se tornaram na prática social cuidadores de idosos.
\end{abstract}

Palavras-chave: representação social, rede social, cuidado do idoso.

\section{Para citar este artículo:}

Maldonado, A., Vizeu, B., Giacomozzi, A., \& Berri, B. (2017). Representações sociais do cuidado ao idoso e mapas de rede social. Liberabit, 23(1), 9-22. doi: 10.24265/liberabit.2017.v23n1.01

\begin{abstract}
This study aimed to understand the social representations of elderly people self-care, care to other elders and their social network, by relating them to care practices used by social networks. The study included a survey distributed to 102 elderly subjects who were asked about their gender and dependency level, in order to identify the elderly social network and the structural part of the social representation of elderly people care. The results shown by the configuration and network map indicate a difference between gender in the elderly social network configuration, where men and independent elderly felt less loneliness, which may reflect a slight deterioration of dependent elderly's social network. In social practice, elderly people care is assigned to informal caregivers, family members and women. The social representation of elderly people care mainly included the pragmatic aspects of care, functionality and passivity. There is a need for educational policies related to health and aging that favor not only the elderly, but also people who are close to experience old age, especially those who have become, in social practice, elderly caregivers.
\end{abstract}

Keywords: social representations, social network, elderly care.

Este es un artículo Open Access bajo la licencia Creative Commons Atribución-NoComercial-CompartirIgual 4.0

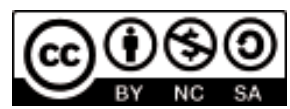




\section{Introdução}

O envelhecimento populacional vem ocorrendo em âmbito mundial, decorrente tanto da queda da mortalidade infantil e fecundidade, quanto do aumento da expectativa de vida (Instituto Brasileiro de Geografia e Estatística [IBGE], 2013; Mendes, Gusmão, Faro, \& Leite, 2005). Projeções indicam que em 2030 o grupo etário dos idosos representará 13.44\% da população brasileira (IBGE, 2013), tal mudança acarreta em uma transição demográfica e epidemiológica, gerando aumento de doenças crônicas e neurodegenerativas, condições de saúde denominadas não-fatais. Ademais, no Brasil e na América Latina, o processo de envelhecimento ocorre num contexto desfavorável, com destaque para pobreza e desigualdades sociais e econômicas (Caldas, 2003; Carrillo-Hernandez \& VazquezGarniza, 2014; Lima-Costa, Barreto, Giatti, \& Uchôa, 2003).

Torna-se necessário, portanto, a implementação de medidas alternativas para o cuidado dos idosos. $\mathrm{O}$ cuidar ocorre com configurações variadas, incluindo hospitais, domicílios, instituições, escolas, comunidades (Caldas, 2006; Diogo \& Duarte, 2006; Duca, Thumé, \& Hallal, 2011). Estudos da representação social do cuidado de idosos apontam para aspectos positivos do cuidado, como importância da manutenção da vida do idoso com comportamentos que expressem amor, carinho e dedicação, ou a partir de práticas diárias, como alimentação, banho e medicação (Lima, 2002), estímulo e aceitação (Souza \& Menezes, 2009). Entretanto, também foram encontrados elementos com conteúdos negativos, como insegurança ao cuidar, medo da incapacidade de cuidar, piedade, culpa, revolta, angústia e raiva (Lima, 2002).

Outras variáveis que interferem no cuidado do idoso dizem, respeito às normas, atitudes, valores e crenças (Battini, Maciel, \& Finato, 2006), o que nesta pesquisa foi estudado por meio das representações sociais (RS), fenômeno que caracteriza uma forma de conhecer o mundo na qual são construídas explicações pautadas no conhecimento do senso comum referente a um objeto social, nesse caso, o cuidado ao idoso. A teoria das RS operacionaliza formas de conhecimento dos objetos, possibilitando acessar sua dinâmica e diversidade (Moscovici, 1978). Essa perspectiva tem sido utilizada para estudar o envelhecimento como processo, a velhice enquanto etapa evolutiva e o idoso, assim como as variáveis aí envolvidas (Martins, Camargo, \& Biasus, 2009; Teixeira, Nascimento-Schulze, \& Camargo, 2002; Torres, 2010; Veloz, Nascimento-Schulze, \& Camargo, 1999).

Ainda, o conjunto de ideias, crenças, atitudes e informações que compõe as representações sociais de um objeto se organizam em torno de uma estrutura e constituem um sistema sociocognitivo. Esses conteúdos se estruturam em elementos hierarquizados em um núcleo central e em torno deste se organizam os elementos periféricos (Abric, 1994; Abric, 1998; Abric, 2001; Abric, 2003), o núcleo central é constituído das cognições que singularizam e dão significados à representação, determinando sua organização interna (Abric, 1998; Sá, 1996). Estudos da representação social do envelhecimento constataram que o pensamento social sobre este objeto se associa a aspectos tanto positivos: sabedoria e experiência (Eiras, 2002; Martins et al., 2009; Torres, 2010; Veloz et al., 1999; Wachelke et al., 2008), quanto negativos: doença, solidão, limitações / dificuldades físicas, incapacidades / perdas físicas e morte (Eiras, 2002; Martins, 2002; Martins et al., 2009; Veloz et al., 1999; Wachelke et al., 2008).

O processo de envelhecimento é alicerçado por inúmeras variáveis que determinam a qualidade do envelhecer. Dentre elas, o apoio recebido pelo idoso advindo de sua rede social é uma condição que propicia melhor qualidade de vida e enfrentamento das perdas, otimizando os ganhos adquiridos com a idade (Battini et al., 2006). Dessa forma, descobrir novos fatos sobre as representações sociais do cuidado do idoso proporciona espaço para o discurso do próprio idoso e de que forma há interação com a 
rede social deste, permitindo auxiliar que outras pessoas construam novos olhares e perspectivas sobre o fenômeno do cuidado, propiciando tanto avanço do conhecimento científico, quanto para a sociedade como um todo.

Por meio dessas considerações, formulou-se a pergunta de pesquisa que norteou esse estudo a partir da investigação de como se constitui o saber social acerca das demandas e cuidados relativos ao idoso: Quais são as representações sociais do cuidado da pessoa idosa para o idoso? Para responder esse questionamento e compreendê-lo de forma efetiva esse estudo teve como objetivos identificar a rede social do idoso, averiguando a existência ou não de um cuidador, realizar a avaliação funcional do idoso para averiguar se o mesmo possuía algum grau de dependência e identificar os elementos que compõem as RS de pessoas idosas referente ao cuidado do idoso.

\section{Método}

A presente pesquisa se caracterizou como um estudo de natureza empírica, realizado por meio de pesquisa de campo. Possuiu delineamento descritivo e comparativo, e foi composta por amostra não probabilística aleatória, por acessibilidade.

Participaram 102 idosos acessados por meio das Unidades Básicas de Saúde (UBS) da Grande Florianópolis, Santa Catarina, sendo 52 homens e 50 mulheres. A média de idade dos mesmos foi de 76 anos e 7 meses ( $\mathrm{DP}=8.37$ ), com idade mínima 65 anos e máxima de 98 anos. Quando divididos por sexo a média de idade permaneceu semelhante à amostra global, com aproximadamente 76 anos para ambos os sexos ( $D P=8.62$ e $D P=8.19$ para homens e mulheres respectivamente).

Os critérios de inclusão na amostra foram (a) idade igual ou acima de 65 anos, conforme designado pela Organização Mundial da Saúde para os países desenvolvidos (Mendes et al., 2005); (b) não ser portador de incapacidades cognitivas, aferido pelo
Instrumento de avaliação sociofuncional em idosos (IASFI; Fonseca \& Rizzotto, 2008); (c) para os participantes com dependência, o grau considerado foi de dependência modificada, com requisição de ajuda entre $25 \%$ e $75 \%$ das tarefas, aferido pelo IASFI (Fonseca \& Rizzotto, 2008); e (d) para os participantes independentes, o grau considerado foi de independência modificada ou completa, sem a necessidade de ajuda para executar as tarefas, aferido pelo mesmo instrumento.

O local utilizado para a coleta de dados foi determinante para acessar tanto idosos dependentes quanto idosos independentes. O posto de saúde foi o local escolhido para a realização da coleta de dados, todavia, a coleta de dados transcorria sem sucesso, e desta maneira, o locus foi modificado para a residência de cada idoso. O procedimento para acessar as UBS iniciou com a solicitação de autorização à Secretaria de Saúde através do envio do projeto de pesquisa, juntamente com o Parecer Consubstanciado N. ${ }^{\circ}$ 2151/13 do Comitê de Êtica em pesquisa da UFSC. Após o envio e reuniões com responsáveis, a Secretaria de Saúde autorizou a realização da pesquisa nas Unidades de Saúde.

Como já mencionado, a melhor estratégia encontrada, após a tentativa de coleta no próprio posto de saúde, foi a visita domiciliar. As primeiras visitas domiciliares foram feitas junto com uma agente de saúde, a qual apresentou a pesquisadora e convidou a pessoa idosa para participar da pesquisa. A aplicação do questionário aconteceu em momento posterior, com agendamento prévio. Após as primeiras visitas domiciliares constatou-se não haver a necessidade de uma visita prévia antes da aplicação do questionário, e, assim, os demais contatos foram realizados por telefone, momento no qual foi explicada a pesquisa e também realizado o convite para a participação. Já com o aceite do participante era feito um agendamento prévio. A duração média das aplicações dos questionários foi 33 minutos. 
Como instrumento para a coleta de dados utilizouse um questionário, constituído pelos seguintes instrumentos: (a) Técnica de Evocação Livre, (b) Adaptação do IASFI (Fonseca \& Rizzoto, 2008), (c) Mapa de Redes e (d) questões referentes as características sociodemográficas e estado de saúde. Para a análise das questões fechadas e as variáveis sociodemográficas foi empregada estatística descritiva: distribuição de frequência, medidas de tendência central, medidas de dispersão, e estatística relacional, por meio do software SPSS (pacote estatístico para as ciências sociais, versão 17).

A adaptação do IASFI (Fonseca \& Rizzotto, 2008) foi composta pelos itens 3 a 33 e realiza uma avaliação a partir das categorias expostas na fundamentação, as quais são: Atividade Básica de Vida Diária, Atividade Instrumental de Vida Diária e a Atividade Avançada de Vida Diária. Os itens componentes de cada categoria, a exceção da última, foram pontuados a partir dos seguintes critérios: (a) necessita de ajuda total para realizar a atividade; ajuda máxima (indivíduo realiza até $25 \%$ da atividade sozinho), (b) ajuda moderada (indivíduo realiza até 50\% da atividade sozinho), (c) ajuda mínima (indivíduo realiza até $75 \%$ da atividade sozinho), (d) só precisa de supervisão para realizar a atividade; realiza a atividade com independência modificada e (e) realiza a atividade com independência completa.

Para a análise do material coletado pela técnica de evocação livre foi utilizado o software Evocation 2000 (Vergès, 1999). Esse programa realiza uma análise lexicográfica, considerando a frequência e a ordem média de evocação, possibilitando identificar a centralidade dos elementos das representações. O IASFI foi pontuado logo após o término da aplicação com cada participante, pois o mesmo propiciou cumprir a composição da amostra do estudo, no que se refere ao grau de dependência versus independência. E para as questões fechadas e as variáveis sociodemográficas foi empregada estatística descritiva: distribuição de frequência, medidas de tendência central (médias, modas e medianas), medidas de dispersão (desvio-padrão), e estatística relacional (Qui-quadrado) por meio do software SPSS (versão 17).

O protocolo desse estudo seguiu os preceitos éticos previstos pela Resolução do Conselho Nacional de Saúde (CNS 196/96) e foi aprovado pelo Comitê Nacional de Ética em Pesquisa com Seres Humanos (CONEP), sob o Parecer Consubstanciado N. ${ }^{\circ} 2151 /$ 13. A presente pesquisa foi apresentada aos participantes, os quais foram informados sobre à condição voluntária de participação, a possibilidade de desistência em qualquer etapa da pesquisa, como também sobre o sigilo que resguardava os mesmos, ainda, todos os participantes assinaram duas vias do Termo de Consentimento Livre e Esclarecido.

\section{Resultados}

Os resultados da caracterização sociodemográfica mostraram que com relação à escolaridade, houve predominância dos participantes que estudaram até o ensino fundamental completo (76.5\%), enquanto $12.7 \%$ estudaram até o ensino médio completo, $4.9 \%$ até o ensino superior completo, $5.9 \%$ analfabetos. Considerando a situação socioeconômica, 70.6\% informou que sua renda consistia de até três salários mínimos, $15.7 \%$ de três a cinco salários, e, $13.7 \%$ mais que cinco salários.

Conforme o instrumento IASFI, 51\% dos participantes não apresentaram grau de dependência, enquanto $35.3 \%$ necessitavam de ajuda em $25 \%$ das atividades de vida diária, 6.9\% careciam de ajuda em $50 \%$ e igual proporção (6.9\%) apresentavam grau de dependência em $75 \%$ das mesmas. Constatou-se maior número de participantes dependentes entre as idades de 75 a 84 anos.

Sobre as atividades avançadas da vida diária, na primeira, viajar sozinho, houve distribuição uniforme entre sexos, com predomínio de pessoas idosas que não viajam sozinhas (68.6\%). Referente a dirigir e realizar atividades manuais, respectivamente $17.6 \%$ e $34.3 \%$ afirmou realizá-las, com predomínio de 
homens para a primeira e mulheres para a segunda. Ambas as associações foram significativas $\left[\chi_{(1)}^{2}=18.04, p<.001\right]$ e $\left[\chi_{(1)}^{2}=4.62, p<.05\right]$, com forças moderada $(\mathrm{C}=.38)$ e fraca $(\mathrm{C}=.20)$.

Em relação ao estado de saúde autodeclarado, os estados "bom" e "regular" predominaram, respectivamente com um total de 42 e 43 frequências. Enquanto para homens o predomínio foi de estado de saúde bom (26), para mulheres houve prevalência de estado de saúde regular (29). Esta associação foi significativa $\left[\chi_{(3)}^{2}=8.33, p<.05\right]$, com força moderada $(V=.28)$. Os problemas de saúde relatados por homens foram, respectivamente: acidente vascular cerebral, queda com fratura do fêmur, enfisema pulmonar, baixa visão e problemas osteo-articulares. Mulheres relataram: acidente vascular cerebral, problemas osteo-articulares, baixa visão, insuficiência cardíaca, doença de chagas, Parkinson, e queda com fratura do fêmur.

A maioria dos participantes informou que é aposentada (86.3\%), e 26.4\% é pensionista. Desses, a maioria são mulheres. Apenas $11.7 \%$ dos participantes referiu trabalhar. Os participantes sem o benefício previdenciário são $13.7 \%$, desses $11.7 \%$ são do sexo feminino. Constatam-se associações significativas entre "sexo" e "ser aposentado" $\left[\chi_{(1)}^{2}=7.83, p<.05\right]$, e "sexo" e "ser pensionista" $\left[\chi_{(1)}^{2}=13.67, p<.001\right]$, destacando-se homens como aposentados, mulheres como pensionistas e com um menor número de benefícios da previdência. As forças destas associações foram moderadas $(C=.26$ e .34$)$.

\section{Composição da rede social}

Verificou-se que 52.9\% residem com o cônjuge, $23.5 \%$ com filhos, $15.7 \%$ sozinhos, $7.8 \%$ com outras pessoas (amigos e/ou vizinhos). Observou-se associação estatisticamente significativa entre "sexo" e "morar com cônjuge" [ $\left.\chi_{(1)}^{2}=17.45, p<.001\right]$, com destaque para homens. Na associação entre "sexo" e "morar sozinho", se destacam mulheres $\left[\chi_{(1)}^{2}=6.95\right.$, $p<.05]$. Ambas forças foram moderadas $(\mathrm{C}=.38$ $\mathrm{e} \mathrm{C}=.25$ ).
Quanto à situação conjugal, 53.3\% dos participantes são casados, 6.9\% solteiros, 3.9\% divorciados/separados, e $35.3 \%$ viúvos. Houve associação significativa entre sexo e situação conjugal, destacando mulheres viúvas e homens casados $\left[\chi_{(3)}^{2}=24.98, p<.001\right]$, com associação moderada $(V=.49)$. Há um maior número de mulheres solteiras que residem sozinhas e de homens casados residindo com cônjuge. Houve associação significativa entre "dependente" e "morar com cônjuge" [ $\left.\chi_{(1)}^{2}=6.95, p<.05\right]$, "dependente" e "morar com filho" $\left[\chi_{(1)}^{2}=8.47, p<.05\right]$, e, "dependente" e "morar com outros" $\left[\chi_{(1)}^{2}=5.46, p<.05\right]$. As três associações obtiveram força moderada $(\mathrm{C}=.24, \mathrm{C}=$ .27 e $\mathrm{C}=.22$ ).

\section{Mapa da rede social}

O Mapa de Redes (Sluzki, 1997) auxiliou na identificação do cuidador, se formal ou informal, bem como a intensidade dos vínculos estabelecidos entre o idoso e a rede. Três questões antecederam à aplicação do Mapa de Redes: (a) Neste momento quais as pessoas mais importantes para o/a senhor/ a? Justifique; (b) Quais são as pessoas que o/a senhor/a tem contato frequente?; e (c) Quais as pessoas que atualmente o ajudam em suas necessidades diárias? Assim foi possível destacar três categorias de compreensão da rede social do idoso: "pessoas importantes", "pessoas com contato frequente" e "pessoas que ajudam/apoiam/cuidam".

Conforme a Figura 1, o quadrante Família se destacou na frequência de citações nas três categorias (283). Em segundo lugar, o quadrante Comunidade/Profissionais citado 60 vezes, dessas, 32 referiam profissionais de saúde, como enfermeiros, agentes comunitários e cuidadores formais, com destaque para os últimos. Com 21 citações, o quadrante "Amizades" ficou em/ terceiro e com 5 citações o quadrante "Relações de trabalho/Grupos, no qual foi citado a participação em grupo de idosos e religiosos. 


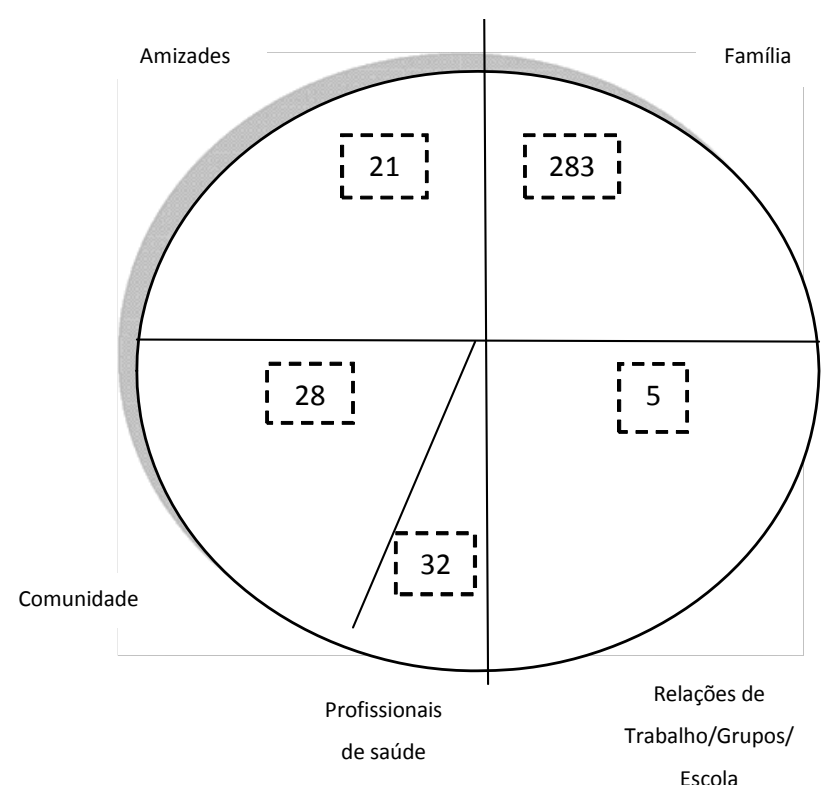

Figura 1. Composição do mapa de redes segundo a frequência de citações por quadrante.

Observou-se que dentre os familiares, os membros que se destacaram como mais importantes para os participantes foram os "filhos" (96 citações). Considerando a amostra global, 29 citaram que as filhas são importantes e 12 citaram que os filhos o são.

Em segundo lugar, os membros com destaque são os cônjuges (43 citações), com destaque para mulheres, com 33 participantes citando esposas como importantes. Houve relação entre ser mulher e prestar apoio ao idoso, com força média de associação entre as variáveis $\left[\chi_{(1)}^{2}=20.99\right.$, $p \leq .001, \mathrm{C}=.47]$. Outros membros citados foram os netos (30 citações), noras/genros (16), irmãos (15), bisnetos e sobrinhos ambos com 9 citações.

Na segunda categoria, "pessoas com contato frequente", o quadrante Família obteve 97 citações. Em seguida, o quadrante Comunidade/Profissionais de saúde (27 citações), as Amizades (13) e Relações de trabalho/Grupo (5). Na terceira categoria, "pessoas que ajudam/apoiam/cuidam", destaca-se o quadrante "Família" com 86 citações. Os quadrantes "Comunidade/Profissionais de Saúde" e Amizades obtiveram, respectivamente, 24 e 3 citações.

Considerando a importância da terceira categoria no escopo desse estudo, "pessoas que ajudam/ apoiam/cuidam", as análises que seguem foram realizadas privilegiando-a. Salienta-se que o número de participantes que citaram cuidadores familiares (86), não corresponde ao número de cuidadores citados (106), já que os participantes poderiam citar mais de um "cuidador". Referente aos citados, 83 das 106 citações de cuidadores foram direcionadas para os "filhos" e "cônjuges", com destaque para os primeiros. Quando os familiares são separados por sexo, a distribuição de frequência se configura dessa forma: filhos (14), filho (13), filha (36), marido (3), esposa (17), neto (2), neta (5), genro (2), nora (5), irmã (5), sobrinha (3), cunhada (1). O sexo feminino sobressai ao masculino com 72 citações contra 34 . No grau de parentesco "filho", 14 citações não discriminaram o sexo, porém, quando o sexo foi discriminado prevaleceu o feminino. 
A terceira categoria, pessoas que apoiam/ajudam/ cuidam, também foi analisada segundo a intensidade do vínculo entre o idoso e os membros da rede. Para tanto, os participantes classificaram seus vínculos em três níveis de proximidade emocional: alto, relações íntimas; médio, contato menos íntimo; e, baixo, relações ocasionais.
Na Figura 2 consta a distribuição da rede social conforme a intensidade do vínculo estabelecido. Observou-se maior concentração de membros no primeiro nível de proximidade, predominando o quadrante familiar.

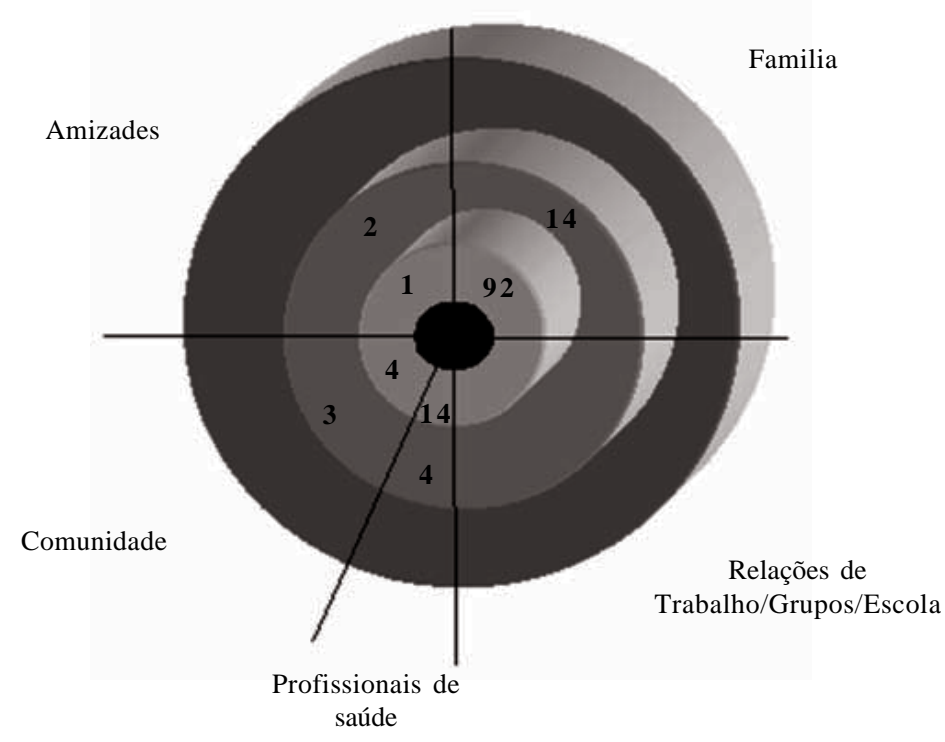

Figura 2. Distribuição dos membros da rede social segundo a intensidade do vínculo com o idoso.

Em todos os quadrantes da Figura 2, quanto menor o grau de intimidade, menor o número de membros. Considerando que a categoria analisada compõe as pessoas que ajudam/auxiliam/cuidam, percebe-se que estas pessoas possuem intimidade com o idoso. Houve tendência em classificar os membros da rede social no primeiro nível de proximidade (111 membros), havendo 23 no segundo nível e nenhum no terceiro. O resultado encontrado é coerente com o tipo de relacionamento estabelecido, já que cuidar/auxiliar/apoiar requer periodicidade, impossibilitando um relacionamento ocasional, como o que se entabula com pessoas que estariam situadas no último nível do mapa.

\section{Análise estrutural da RS do cuidar da pessoa idosa}

A constituição da estrutura das representações sociais de "cuidar da pessoa idosa" decorreu da análise lexicográfica com as palavras evocadas. O software Evocation 2000 (Vergès, 1999) considera dois critérios, a frequência e a ordem média de evocação, distribuindo a localização dos elementos (palavras) segundo este duplo critério. Assim, os elementos com maior frequência e evocados primeiramente possuem maior probabilidade de pertencerem ao núcleo central, enquanto os elementos com menor frequência e evocados tardiamente constituem a periferia da representação.

Na análise lexicográfica obteve-se 486 evocações, dessas 127 eram palavras diferentes, sendo 
consideradas aquelas com frequência superior a 5. A frequência intermediária foi 15 e a ordem média de evocação 3. Pode-se observar na Tabela 1, no quadrante superior esquerdo destacam-se os elementos "alimentação", "carinho", "higiene", "respeito", "atenção" e "ser cuidado".

Tabela 1

Diagrama de quadrantes da RS de cuidar da pessoa idosa

\begin{tabular}{|c|c|c|c|c|c|c|}
\hline & \multicolumn{3}{|c|}{$\mathrm{OME}<3$} & \multicolumn{3}{|c|}{$\mathrm{OME} \leq 3$} \\
\hline & Elemento & $\mathrm{F}^{*}$ & OME** & Elemento & $\mathrm{F}$ & OME \\
\hline \multirow{7}{*}{$f \geq 15$} & Alimentação & 34 & 2.76 & Medicamento & 23 & 3.52 \\
\hline & Carinho & 28 & 2.46 & Familia & 22 & 3.36 \\
\hline & Higiene & 26 & 2.84 & Acompanhamento & 16 & 3.32 \\
\hline & Respeito & 20 & 2.50 & & & \\
\hline & Atenção & 19 & 2.52 & & & \\
\hline & Ser_cuidado & 19 & 1.84 & & & \\
\hline & Zelo & 15 & 1.86 & & & \\
\hline \multirow{8}{*}{$\mathrm{f}<15$} & Amor & 14 & 2.71 & Assistência & 10 & 3.00 \\
\hline & Ajuda & 13 & 2.92 & Atividade_física & 7 & 3.42 \\
\hline & Cuidar_saúde & 13 & 2.46 & Educação & 7 & 3.42 \\
\hline & Autocuidado & 10 & 1.80 & Apoio & 6 & 3.83 \\
\hline & Cuidar_queda & 9 & 2.77 & Conversar & 5 & 3.80 \\
\hline & Saúde & 9 & 2.77 & & & \\
\hline & Paciência & 8 & 2.87 & & & \\
\hline & Dificuldade & 6 & 2.16 & & & \\
\hline
\end{tabular}

Nota: *F = frequência de evocação. ${ }^{* *} \mathrm{OME}=$ Ordem Média de Evocação.

Estes elementos possuem tendência de centralidade, possivelmente constituindo o núcleo central do cuidado ao idoso. Os elementos "alimentação" e "higiene" indicam uma representação permeada por aspectos pragmáticos do cuidado. Esta representação também é composta por elementos afetivos, como "carinho", "atenção", "respeito" e "zelo", sugerindo comportamentos desejáveis no cuidar. O elemento "ser cuidado", não obstante ter uma frequência menor de evocação (19) possui uma maior ativação (OME = 1.84) em comparação aos demais, a exceção de "zelo", que expressa a necessidade de receber cuidados, e também os tipos de cuidados, como pela via medicamentosa, e o dispensado pela rede social do idoso, com o elemento "família" e "acompanhante". A partir do teste de centralidade somente o elemento "higiene" não foi confirmado como central na representação.

No quadrante inferior esquerdo, ou zona de contraste, evidencia-se o cuidado com a saúde, com as quedas e o autocuidado. Esse último, apesar da menor frequência de evocação, apresenta-se como elemento importante para os participantes porque sua ordem média de evocação esteve entre as primeiras posições. O elemento "amor" é evocado entre a segunda e terceira posições com frequência próxima aos elementos centrais, o que salienta relevância juntamente com os demais elementos afetivos da representação. 
Na segunda periferia, quadrante inferior direito, elementos diversos assinalam que no cuidado ao idoso a "assistência", "educação", "apoio" e "conversar" são importantes. A "atividade física" ganha relevo para a periferia da representação.

Ao comparar os elementos por grupos, pessoas idosas dependentes versus independentes, e homens versus mulheres, verifica-se que os elementos "ser cuidado" $\left[\chi_{(1)}^{2}=4.85, p<.05\right]$ e "família" $\left[\chi_{(1)}^{2}=10.46 p<.001\right]$ se destacam para os idosos dependentes, com força moderada $(\mathrm{C}=.25)$ e $(\mathrm{C}=.43)$. O elemento "cuidar saúde" possui maior relevância para idosos independentes, com conotação preventiva $\left[\chi_{(1)}^{2}=3.62, p<.05\right]$, com força de associação moderada $(\mathrm{C}=.22)$. Por fim, os elementos "amor" $\left[\chi_{(1)}^{2}=9.53, p<.001\right]$ e "atenção" $\left[\chi_{(1)}^{2}=11.04, p<.001\right]$ ganham relevância para o sexo feminino, com forças de associação moderadas $(\mathrm{C}=.35)$ e $(\mathrm{C}=.38)$.

\section{Discussão}

Este estudo procurou identificar as representações sociais do cuidado à pessoa idosa, bem como a rede social de 102 pessoas com mais de 65 anos. Avaliouse também o grau de dependência dos participantes de acordo com o instrumento IASFI.

Quanto à dependência, houve predomínio do primeiro grau, ou seja, os participantes precisam de ajuda em até $25 \%$ das tarefas. Esse grau marca que o idoso, mesmo recebendo ajuda em atividades diárias, possui potencial para gerir sua vida, tendo autonomia, que é a habilidade para pensar, agir e avaliar as situações da vida segundo o seu próprio ponto de vista, implicando em escolhas e decisões. Autonomia também é uma variável que auxilia no processo de envelhecimento (Battini et al., 2006), e se relaciona a modos individuais de agir conforme suas próprias regras (World Health Organization, 2005).

Com relação aos problemas de saúde dos participantes, o AVC foi predominante para ambos os sexos, porém as quedas e o enfisema pulmonar foram prevalentes para homens, e os problemas osteo-articulares para mulheres. E enquanto homens declararam que seu estado de saúde é bom, as mulheres o declararam como regular. Além da autodeclaração do estado de saúde, a declaração de doenças também se diferenciou entre os sexos, com predomínio de mulheres respondendo afirmativamente para alguma morbidade. Diversas pesquisas constataram um maior número de relato de morbidades para o sexo feminino (Barros, Zanchetta, Moura, \& Malta, 2009; Brito \& Camargo, 2011; Fonseca, Blank, Barros, \& Nahas, 2008). Em contrapartida, o sexo masculino, demonstra-se mais predisposto aos cuidados à saúde em caso de agravos e ou morbidade (Brito \& Camargo, 2011). Ainda, os dados corroboram com um estudo o qual verificou que os homens tendiam a ter uma posição mais positiva de sua enfermidade, e também acreditavam ter mais controle sobre a mesma, apresentando ainda uma percepção de maior apoio familiar a sua condição (Ofman, Pereyra, Cófreces, \& Stefani, 2015).

Sobre a escolaridade, apesar da amostra global da pesquisa declarar com preponderância o ensino fundamental incompleto, constatou-se que a maioria desses apresenta quadro de analfabetismo funcional, ou seja, são pessoas que mesmo capacitadas para decodificar letras, frases e pequenos escritos, não consegue interpretar textos e fazer cálculos. Conforme dados do IBGE (2013) dentre os grupos de maior incidência de analfabetismo estão as pessoas acima de 65 anos, com analfabetismo funcional. Há ainda um progressivo envelhecimento da população analfabeta, o que dificulta a modificação do quadro devido à dificuldade de acesso ao ensino. É possível que a baixa escolaridade dos participantes possa ter interferido nos resultados encontrados na categoria "atividades avançadas de vida diária" representada pelas atividades de viajar e dirigir, pois a proporção de participantes que não realizam essas atividades sozinhos foi elevada. Tais resultados do perfil sociodemográfico também foram encontrados em outro estudo (Victor, Ximenes, 
Almeida, \& Vasconcelos, 2009), que verificou que os idosos possuem em geral baixos salários, o que pode ser um fator limitador no acesso a bens de serviço e de consumo, tais como, moradia e alimentação. Quanto à escolaridade, esses autores encontraram um número elevado de analfabetismo declarado.

Sobre a rede social, predominaram os participantes que moram com alguém, destacandose cônjuges. Isso foi prevalente para os homens, o que os posicionou em condição de menor solidão e maior apoio. $\mathrm{O}$ apoio é um indicador de qualidade de vida ao longo do envelhecimento, facilitando o enfrentamento de perdas e a otimização dos ganhos (Battini et al., 2006). Esses resultados indicam também uma diferenciação na situação conjugal dos participantes, já que prevaleceram homens casados e mulheres viúvas. A prevalência do número de mulheres viúvas, em parte, pode estar ligada ao fato de que os homens possuem maior dificuldade em aderir comportamentos de saúde, enquanto mulheres se engajam mais facilmente em comportamentos saudáveis, evitando comportamentos de riscos, como o hábito de beber (Brito \& Camargo, 2011; Matos \& Souza-Albuquerque, 2006).

Sluzki (1997) estabelece relação entre a rede social e a saúde apontando que existe uma relação bidirecional entre ambas, afetando-se reciprocamente. Essa relação ganha relevo no caso dos idosos dependentes, já que em situações de agravo à saúde pode ocorrer o que o autor denomina de deterioração recíproca. Alguns impactos nas redes sociais em decorrência dos processos de adoecimento são: a deterioração da qualidade, a redução do tamanho e a impossibilidade de acesso a membros distantes geograficamente.

Foram investigadas três categorias referentes à rede social de apoio ao idoso: "pessoas importantes", "pessoas com contato frequente" e "pessoas que ajudam/apoiam/cuidam". Verificou-se que o quadrante família predominou nas três categorias, respectivamente com 100, 97 e 86 citações. Esse resultado tem sua origem relacionada a vários aspectos, e um deles é o critério de avaliação dos membros que entram nessas categorias. Enquanto na primeira o critério foi a vinculação sanguínea, na segunda categoria foi haver uma relação concreta de apoio. A rede social e a saúde estabelecem uma relação bidirecional, o que significa afirmar que tanto a constituição da rede social pode afetar a saúde, quanto o inverso, segundo Sluzki (1997). Nos casos de agravos à saúde pode ocorrer o que o autor denomina de deterioração recíproca, impacto negativo na rede social devido a quadros de dependência, que pode ser um aspecto relevante que possivelmente justifica a diminuição de membros que compõem a última categoria. Estudo de Erbolato (2006) também verificou predomínio dos familiares como principais integrantes da rede social do idoso, entendendo-se a família tanto nuclear quanto ampliada.

A partir do questionamento "com quem reside", observou-se prevalência do cônjuge como o principal acompanhante, porém, quando questionados sobre as pessoas importantes de sua vida, os filhos se destacam em primeiro lugar, e em segundo os cônjuges. O mesmo ocorre na categoria referente às "pessoas que ajudam/apoiam/cuidam", com vantagem dos filhos em detrimento aos cônjuges. Esses resultados indicam que compor a rede social significativa da pessoa idosa não prediz o cuidado dispensado a ela, já que o membro apontado como importante não necessariamente possui proximidade física ou cuida do idoso.

A análise estrutural das representações sociais de "cuidar da pessoa idosa" demonstrou que as palavras que constituíram os possíveis elementos centrais foram "alimentação, carinho, respeito, atenção, ser cuidado e zelo". A primazia hierárquica do elemento "alimentação" aponta para destaque dos aspectos pragmáticos do cuidado em detrimento aos aspectos afetivos, representado principalmente pelo elemento "carinho", o segundo em hierarquia, já que foi um dos elementos mais prontamente evocados. 
A representação social é um tipo de conhecimento estruturado e possui um papel determinante na forma como os indivíduos reagem face à realidade (Campos \& Rouquette, 2003), o que implica afirmar que para os participantes dessa pesquisa a representação de cuidar do idoso está relacionada com as práticas de cuidar. Desse modo, o elemento mais central dessa representação traz como característica principal a funcionalidade.

Os demais elementos que compuseram o núcleo central (respeito, atenção, ser cuidado e zelo) ressaltam aspectos afetivos no quesito cuidar, porém igualmente vinculados à prática de cuidado, já que ensejam comportamentos desejáveis ao cuidador e no relacionamento com a pessoa cuidada. Esses elementos também foram confirmados como centrais da representação social de cuidar do idoso. Além disso, o elemento "ser cuidado" denota que os participantes além de conceberem que o idoso possui necessidade de receber cuidados, também o caracterizam como passivo, desconsiderando o autocuidado no processo de envelhecimento. Em resumo, os cuidados devem ser dispensados ao idoso, possivelmente por outra pessoa de sua rede social. Os comportamentos que se enquadram na categoria autocuidado são essenciais para a independência e a autonomia também no processo de envelhecimento, o que significa cuidar e se responsabilizar pela sua saúde e demais esferas da vida (Caldas, 2006).

Em relação ao sexo, os elementos evocados com predomínio pelas mulheres foram "amor e atenção", o que caracteriza uma representação feminina com predominância de aspectos afetivos, diferentemente dos elementos salientados pela amostra global. Somente o elemento "atenção" apresentou centralidade nessa representação. O elemento "amor" constituiu a periferia, devido a sua baixa ordem de evocação.

Em relação à dependência, os elementos que ganharam destaque para os participantes idosos dependentes foram "ser cuidado e "família". O primeiro elemento indica uma marca de passividade, como discutido anteriormente, enquanto o segundo reitera a importância dos membros familiares no processo de cuidado, principalmente quando existe a necessidade de cuidados de saúde. Entretanto, apesar da saliência que o elemento "ser cuidado" ganha para o grupo de pessoas idosas dependentes, esse elemento compôs o núcleo dessa representação para a amostra global de participantes.

Os elementos periféricos "medicamento, família e acompanhamento" possuem um papel complementar e específico em relação ao núcleo central (Abric, 2003). Os elementos da periferia próxima confirmam a inter-relação com os elementos centrais da representação. Quando o elemento "ser cuidado" ganha relevo no núcleo central, a periferia especifica de que maneira os participantes compreendem esse cuidado, a partir do uso de medicamentos, com o apoio da família ou com algum acompanhante.

Outros elementos que indicam a periferia e são elementos intermediários estão localizados no quadrante inferior esquerdo, tais como: "amor, ajuda, cuidar da saúde, autocuidado, cuidar das quedas, saúde, paciência e dificuldade". Esses elementos foram evocados com baixa frequência, porém com grande prontidão, indicando contextos particularizados da representação de cuidar do idoso. O elemento "cuidar da saúde" se destaca para os participantes idosos independentes. Especula-se nesse sentido que a ação de cuidar da saúde é ainda eminente para as pessoas que não possuem prejuízo na mesma, já que foi um elemento relevante para um grupo circunscrito. O sistema periférico responde por três funções principais, uma delas a concretização (Abric, 1998), isto é, elementos que auxiliam na materialização e entendimento do significado da representação. Os termos "cuidar das quedas" e "cuidar da saúde" evidenciam essa materialização do cuidado do idoso. O sistema periférico possui também as funções de regulação e de defesa. 
Os elementos "assistência, atividade física, educação, apoio e conversar" compuseram o quadrante inferior direito, com evocações menos frequentes e com menor prontidão, ou seja, foram elementos evocados com menor prontidão e demarcam contextos específicos da presente representação. Esses elementos devido à distância do núcleo central são os menos expressivos para a significação do conhecimento social de "cuidar da pessoa idosa". O autocuidado é um cuidado primário e como tal contribui para o desenvolvimento humano e reforça a participação ativa das pessoas em seu próprio cuidado, auxiliando na dinâmica de vida de cada um. Sendo assim, esse deve ser incentivado, pois o desenvolvimento do potencial da pessoa irá incentivar na manutenção da saúde funcional e na autonomia do idoso.

Algumas limitações deste estudo são apontadas até mesmo para dar indicações para estudos futuros. A presente tese utilizou como variáveis o "sexo" e o "grau de dependência", porém não foi possível controlar a última variável, o que pode ter influenciado os conteúdos representacionais. A literatura aponta que o grau de dependência é diferenciador do tipo de cuidado dispendido, do tempo investido, das demandas requeridas, e do desgaste do cuidador; sendo assim, hipotetiza-se que as representações sociais de idosos com grau de dependência maior se diferenciarão de idosos sem nenhum ou pequeno grau de dependência. Para buscar confirmar esse questionamento é necessário um estudo que controle com rigor esta variável e aumente o número da população alvo do estudo.

\section{Referências}

Abric, J. C. (1994). Pratiques e representations sociales. Paris: PUF.

Abric, J. C. (1998). A abordagem estrutural das representações sociais. In A. S. P. Moreira \& D. C. Oliveira (Orgs.), Estudos interdisciplinares de representação social (pp. 27-38). Goiânia: AB.

Abric, J. C. (2001). A abordagem estrutural das representações sociais. In A. S. P. Moreira \& D. C. Oliveira (Orgs.), Estudos interdisciplinares de representação social (pp. 27-38). Goiânia: AB.

Abric, J. C. (2003). Abordagem estrutural das representações sociais: Desenvolvimentos recentes. In P. H. S. Campos \& M. C. S. Loureiro (Orgs.). Representações sociais e práticas educativas (pp. 3757). Goiânia: Ed. da Universidade Católica de Goiás.

Barros, M. B. de A., Zanchetta, L. M., Moura, E. C., de, \& Malta, D. C. (2009). Auto-avaliação da saúde e fatores associados. Revista de Saúde Pública, 43(2), 27-37.

Battini, E., Maciel, E. M., \& Finato, M. da S. S. (2006). Identificação de variáveis que afetam o envelhecimento: análise comportamental de um caso clínico. Estudos de Psicologia, 23(4), 455-462.

Brito, A. M. M., \& Camargo, B. V. (2011). Representações sociais, crenças e comportamentos de saúde: um estudo comparativo entre homens e mulheres. Temas em Psicologia, 19(1), 283-303.

Caldas, C. P. (2003). Envelhecimento com dependência: responsabilidades e demandas da família. Caderno de Saúde Pública, 19(3), 773-781.

Caldas, C. P. (2006). O autocuidado na velhice. In E. V. Freitas, L. Py, F. A. X. Cançado, J. Doll \& M. L. Gorzoni, Tratado de geriatria e gerontologia (pp. 1430-34). Rio de Janeiro: Guanabara Koogan.

Campos, P. H. F., \& Rouquette, M. L. (2003). Abordagem estrutural e componente afetivo das representações sociais. Psicologia: Reflexão e Crítica, 16(3), 435-445.

Carrillo-Hernandez, E., \& Vazquez-Garniza, E. K. (2014). Emociones de ancianos beneficiarios de programas sociales en metrópoli de Guadalajara. Cadernos de Pesquisa, 44(152), 334-353. 
Diogo, M. J. D., \& Duarte, Y. A. O. (2006). Cuidados em domicílio: conceitos e práticas. In E. V. Freitas, L. Py, F. A. X. Cançado, J. Doll \& M. L. Gorzoni, Tratado de geriatria e gerontologia (pp. 3-17). Rio de Janeiro: Guanabara Koogan.

Duca, G. F. del., Thumé, E., \& Hallal, P. C. (2011). Prevalência e fatores associados ao cuidado domiciliar a idosos. Revista de Saúde Pública, 45(1), 113-120.

Eiras, N. (2002). Representações sociais da velhice em instituições públicas de saúde. Revista de Ciências Humanas, Florianópolis, EDUFSC, especial temática, 117-131.

Erbolato, R. M. P. L. (2006). Relações sociais na velhice. In V. Freitas, L. Py, F. A. X Cançado, J. Doll \& M. L. Gorzoni, Tratado de geriatria e gerontologia (pp. 1324-31). Rio de Janeiro: Guanabara Koogan.

Fonseca, F. B. da, \& Rizzotto, M. L. F. (2008). Construção de instrumento para avaliação sócio-funcional em idosos. Texto e Contexto Enfermagem, 17(2), 365-373.

Fonseca, S. A., Blank, V. L. G., Barros, M. V. G. de, \& Nahas, M. V. (2008). Percepção de saúde e fatores associados em industriários de Santa Catarina, Brasil. Cadernos de Saúde Pública, 24(3), 567-576.

Instituto Brasileiro de Geografia e Estatística. (2013). Síntese de indicadores sociais: Uma análise das condições de vida da população brasileira. Recuperado em http://biblioteca.ibge.gov.br/ visualizacao/livros/liv66777.pdf

Lima, C. F. da M. (2002). O Cuidado domiciliar ao idoso com doença de Alzheimer: representações de familiares cuidadores (Dissertação de Mestrado). Universidade Federal da Bahia, Salvador.

Lima-Costa, M. F., Barreto, S., Giatti, L., \& Uchôa, E. (2003). Desigualdade social e saúde entre idosos brasileiros: um estudo baseado na Pesquisa Nacional por Amostras de Domicílios. Caderno de Saúde Pública, 19(3), 745-757.

Martins, C. R. M. (2002). O envelhecer segundo adolescentes, adultos e idosos usuários do SESC Maringá: um estudo de representações sociais (Dissertação de Mestrado). Universidade Federal de Santa Catarina, Florianópolis.
Martins, C. R. M., Camargo, B. V., \& Biasus, F. (2009). Representações sociais do idoso e da velhice para diferentes faixas etárias. Universitas Psychologica, 8(3), 831-847. Recuperado de http:// revistas.javeriana.edu.co/index.php/revPsycho/article/ viewFile388

Matos, A. P. S. de, \& Souza-Albuquerque, C. M. de. (2006). Estilo de vida, percepção de saúde e estado de saúde em estudantes universitários portugueses: influência da área de formação. International Journal of Clinical and Health Psychology, 6(3), 647-663.

Mendes, M. R. S. S. B., Gusmão, J. L. de, Faro, A. C. M. e, \& Leite, R. de C. B. de O. (2005). A situação social do idoso no Brasil: uma breve consideração. Acta Paulista de Enfermagem, 18(4), 422-426.

Moscovici, S. (1978). A representação social da psicanálise. Rio de Janeiro: Zahar.

Ofman, S. D., Pereyra, C. I., Cófreces, P., \& Stefani, D. (2015). Estudio de las representaciones sociales de la hipertensión arterial según género. Liberabit, 21(1), 5970.

Sá, C. P. de (1996). Núcleo central das representações sociais. Petrópolis: Vozes.

Sluzki, C. E. (1997). A rede social na prática sistêmica. Alternativas terapêuticas. São Paulo: Casa do Psicólogo.

Souza, A. dos S., \& Menezes, M. do R. de. (2009). Estrutura da representação social do cuidado familiar com idosos hipertensos. Revista Brasileira de Geriatria e Gerontologia, 12(1), 87-102.

Teixeira, M. C. T. V., Nascimento-Schulze, C. M., \& Camargo, B. V. (2002). Representações sociais sobre a saúde na velhice: um diagnóstico psicossocial na Rede Básica de Saúde. Estudos de Psicologia, 7(2), 351-359.

Torres, T. de L. (2010). Pensamento social sobre envelhecimento, idoso e rejuvenescimento para diferentes grupos etários (Tese de doutorado). Universidade Federal de Santa Catarina, Florianópolis.

Veloz, M. C. T., Nascimento-Schulze, C. M., \& Camargo, B. V. (1999). Representações sociais do envelhecimento. Psicologia: Reflexão e Crítica, 12(2), 479-501. 
Vergès, P. (2000). Esemble de programmes permettant l'analyse des évocations: manuel version 2. Aix-enProvence: LAMES.

Victor, J. F., Ximenes, L. B., Almeida, P. C. de, \& Vasconcelos, F. de F. (2009). Perfil sociodemográfico e clínico de idosos atendidos em Unidade Básica de Saúde da Família. Acta Paulista de Enfermagem, 22(1), 49-54.
Wachelke, J. F. R., Camargo, B. V., Hazan, J. V., Soares, D. R., Oliveira, L. T. P., \& Reynaud, P. D. (2008). Princípios organizadores da representação social do envelhecimento: dados coletados via internet. Estudos de Psicologia, 13(2), 107-116.

World Health Organization. (2005). Organização Mundial de Saúde. Envelhecimento ativo: uma política de saúde (Trad. S. Gontijo). Brasília: Organização PanAmericana da Saúde.

\section{Annie Mehes Maldonado Brito}

Profesora y investigadora de Psicología en la UNIPAMPA - Universidade Federal do Pampa. Doctora y Maestra por el Programa de Pós-graduação em Psicologia - Universidade Federal de Santa Catarina - UFSC.

* mehesm@yahoo.com.br

\section{Andréia Isabel Giacomozzi}

Profesora Adjunta del Departamento de Psicologia y del Programa de Pós-graduação em Psicologia de la Universidade Federal de Santa Catarina. Doctora y Maestra por el Programa de Pós-graduação em Psicologia - UFSC.

agiacomozzi@hotmail.com

\section{Bruna Berri}

Psicóloga por la Universidade Federal de Santa Catarina (2016). Mestranda en Psicología por el Programa de Pós Graduação de la Universidade Federal de Santa Catarina, em la área de Salud y Desarrollo Psicológico.

brunaberri@hotmail.com

\section{Brigido Vizeu Camargo}

Profesor Titular de la Universidade Federal de Santa Catarina, post-doctorado en Psicología Social por el «Institut Interdisciplinaire d’Anthropologie du Contemporaine de l’École des Hautes Études en Sciences Sociales» y miembro fundador del Laboratório de Psicologia Social da Comunicação e da Cognição.

brigido.camargo@yahoo.com.br 